DOI: 10.17516/1997-1397-2021-14-2-230-241

УДК 519.21

\title{
Joint Distribution of the Number of Vertices and the Area of Convex Hulls Generated by a Uniform Distribution in a Convex Polygon
}

\author{
Isakjan M. Khamdamov* \\ National University of Uzbekistan named after Mirzo Ulugbek \\ Tashkent, Uzbekistan \\ Zoya S. Chay ${ }^{\dagger}$ \\ Tashkent University of Information Technologies named after M. al-Khwarizmi \\ Tashkent, Uzbekistan
}

Received 05.07.2020, received in revised form 13.12.2020, accepted 20.01.2021

\begin{abstract}
A convex hull generated by a sample uniformly distributed on the plane is considered in the case when the support of a distribution is a convex polygon. A central limit theorem is proved for the joint distribution of the number of vertices and the area of a convex hull using the Poisson approximation of binomial point processes near the boundary of the support of distribution. Here we apply the results on the joint distribution of the number of vertices and the area of convex hulls generated by the Poisson distribution given in [6]. From the result obtained in the present paper, in particular, follow the results given in $[3,7]$, when the support is a convex polygon and the convex hull is generated by a homogeneous Poisson point process.
\end{abstract}

Keywords: convex hull, convex polygon, Poisson point process, binomial point process, central limit theorem.

Citation: I.M. Khamdamov, Z.S. Chay, Joint Distribution of the Number of Vertices and the Area of Convex Hulls Generated by a Uniform Distribution in a Convex Polygon, J. Sib. Fed. Univ. Math. Phys., 2021, 14(2), 230-241. DOI: 10.17516/1997-1397-2021-14-2-230-241.

\section{Introduction}

This paper is devoted to the study of properties of convex hulls generated by independent observations over a random vector that has a uniform distribution in a convex polygon. Convex hulls are very complex objects from the analytic point of view. Therefore, studying the properties of the simplest functionals of convex hulls, such as, the number of vertices or the area, is not an easy task. This explains the fact that, prior to obtaining the central limit theorem for the number of vertices of a convex hull by P. Groeneboom, the main achievement was considered to be the study of asymptotic expressions for the mean values of similar functionals (see, for example, $[4,5,16])$; the problems on asymptotic expressions for the variance remained unsolved until the appearance of the studies by C. Buchta [1,2] and J. Pardon [14, 15].

It should be noted that P. Groeneboom, using the well-known property of homogeneous binomial point processes, which is that near the boundary of the support, it is almost indistinguishable

\footnotetext{
${ }^{*}$ khamdamov.isakjan@gmail.com

$\dagger$ 'chay1526@mail.ru

(C) Siberian Federal University. All rights reserved
} 
from a homogeneous Poisson point process, and using such powerful techniques as strongly mixing stationary processes and martingales, has proved the central limit theorems for the number of vertices of a convex hull in the case when the support of the original uniform distribution is either a convex polygon or a unit disk. The modified P. Groeneboom technique was applied in [3] to prove limit theorems for the area and perimeter of a convex hull in a polygon, and in [9], to prove a limit theorem for an area outside a convex hull in a disk.

Similar results were obtained later by J.Pardon [16,17] without imposing any regularity conditions on the support boundary. In the present work, there is no need for using martingales, strongly mixing stationary processes, etc.; the approach used is a modification of the methods presented in [7, 10-13]. The results obtained by Sh. K. Formanov, I. M. Khamdamov in [6], are applied here; a joint limit distribution for the number of vertices and the area of the convex hull generated by a Poisson point process in a cone was obtained by elementary analytical and direct probabilistic methods.

\section{Statement of problem and results}

Let $\vec{x}_{j}, j=1,2, \ldots, n$ be the independent observations over a random vector having a uniform distribution in a convex polygon $A$ with $r$ sides. A matrix $X_{n}$ is called a sample, the j-row of which is formed by the components of the vector $\vec{x}_{j}$. Let us denote the convex hull generated by vectors $\vec{x}_{j}, j=1,2, \ldots, n$ by $C_{n}=C_{n}\left(X_{n}\right)$.

We are interested in the joint limit distribution of the following functionals of $C_{n}$ : the total number of vertices $\nu_{n}$ and the area $S_{n}$. It is clear that $C_{n}$, and, consequently, the indicated functionals, are uniquely determined by the set of vertices $W_{n}$. If the principle of vertex labeling is chosen, then it can be represented as a $\nu_{n} \times 2$ matrix. It is easy to show that this matrix has the property of sufficiency with respect to the boundary of the set $A$ - the support of original distribution. The latter circumstance is of interest from the point of view of statistics of uniform distributions.

Before formulating the main results, we introduce some notation. Let $S$ be the area of the polygon $A$. Then we assume that

$$
D_{n}=S-S_{n}
$$

and let

$$
a_{n}=\frac{2 r \log n}{3}, \quad a_{n}^{\prime}=\frac{S a_{n}}{n}, \quad b_{n}=\sqrt{\frac{27}{10 r \log n}}, \quad b_{n}^{\prime}=n \sqrt{\frac{27}{28 r S^{2} \log n}}=\frac{\sqrt{5} n b_{n}}{\sqrt{14} S} .
$$

We denote by $\omega$ a vector having a two-dimensional normal distribution with a zero vector of mean values, unit variances and a correlation coefficient $\sqrt{5 / 14}$.

Let us state the main theorem.

Theorem 1. Under our assumptions, a random vector with components $b_{n}\left(\nu_{n}-a_{n}\right)$ and $b_{n}^{\prime}\left(D_{n}-a_{n}^{\prime}\right)$ converges in distribution to $\omega$.

Let us make the necessary explanations of the notation. The symbols $\stackrel{d}{\Rightarrow}, \stackrel{p}{\rightarrow}, \rightarrow$ a.s. denote convergence in distribution, in probability, and almost sure, respectively. $f(\varepsilon) \asymp g(\varepsilon)$ means that there are positive constants $c_{1}, c_{2}, \varepsilon_{0}$ such that $c_{1} f(\varepsilon) \leqslant g(\varepsilon) \leqslant c_{2} f(\varepsilon)$ for any $0<\varepsilon<\varepsilon_{0}$. Generally $o_{p}(1)$ is used for a sequence of random variables converging in probability to zero. The notation $\xi_{n}=O_{p}(1)$ means that $\sup _{n \geqslant 1} P\left(\left|\xi_{n}\right|>t\right) \rightarrow 0$ as $t \rightarrow \infty$. Everywhere $c, c_{1}, c_{2}, \ldots$ are the positive constants whose values might be changed from line to line and $c(\beta), c_{1}(\beta), c_{2}(\beta)$ 
are the positive constants, depending on the specified arguments. Further, $\xi \stackrel{\text { dis }}{=} \zeta$ means that the random variables $\xi$ and $\zeta$ have a common law of probability distribution.

\section{The Poisson approximation}

In this section, we present the key idea of [7] about the Poisson approximation of a homogeneous binomial point process (h.b.p.p) $B_{n}(A)$ generated by $n$ independent observations of a random variable having a uniform distribution with support $A$ in a slightly different way. Here we consider the more general case, assuming that $A$ is an arbitrary bounded convex set in $R^{2}$.

Let $\Gamma_{A}$ be the boundary of the set $A$. For each $z \in \Gamma_{A}$, consider an open sphere $S(z, \varepsilon)$ of radius $\varepsilon$ centered in $z$. It is easy to see that the set $A_{\varepsilon}=A-\bigcup_{z \in \Gamma_{A}} S(z, \varepsilon)$ is a strip along the border $\Gamma_{A}$. Let us denote $B_{\varepsilon}=A-A_{\varepsilon}$ and assume that $\lambda(A)=1$, where $\lambda(\cdot)$ is the Lebesgue measure.

Let $W_{n}$, as before, be the set of vertices of the convex hull $C_{n}$ generated by $B_{n}(A)$. The next lemma is a simple modification of Lemma 2.1 and its Corollary 2.1 given in [7].

Lemma 1. There is a sequence of positive numbers $\varepsilon_{n}$ converging to zero such that the probability that at least one of the vertices $C_{n}$ laying in $B_{\varepsilon}$, converges to zero in $\varepsilon>\varepsilon_{n}$.

Proof. It is easy to see that the event $E=\left\{W_{n} \cap B_{\varepsilon} \neq \varnothing\right\}$ coincides with the event "there is a pair of neighboring vertices $w_{1}$ and $w_{2}$ such that $w_{1} \in B_{\varepsilon}$ ". Let the straight line $\left(p, z-w_{1}\right)=0$ pass through the point $w_{2}$. Since $w_{1} \in B_{\varepsilon}$, then this line divides $A$ into two parts, the measure of each is no less than some value of $c(\varepsilon)>0$ such that $\lim _{\varepsilon \rightarrow 0} c(\varepsilon)=0$. Therefore at $n>2$

$$
P(E)=\frac{n(n-1)}{2} \iint_{w_{1} \in B_{\varepsilon}, w_{2} \in A} P^{n-2}\left\{n-2 \text { the sample points } X_{n}\right. \text { lie on one side }
$$

$$
\text { of the straight line } \left.\left(p, z-w_{1}\right)=0\right\} d w_{1} d w_{2} \leqslant n^{2}(1-c(\varepsilon))^{n} \text {. }
$$

It remains to assume that

$$
\varepsilon_{n}=\inf \left\{\varepsilon: c(\varepsilon) \geqslant \frac{3 \log n}{n}\right\}
$$

The lemma is proved.

Note that the rate of decrease $c(\varepsilon)$ at $\varepsilon \rightarrow 0$ depends on the smoothness $\Gamma_{A}$. In particular, if $A$ is a sphere, then $c(\varepsilon) \asymp \varepsilon^{\frac{3}{2}}$; if $A$ is a polygon, then $c(\varepsilon) \asymp \varepsilon^{2}$ and etc.

Since we are not interested in the estimates of the rate of convergence in the theorems given below, we will not worry about optimizing the choice of the strip containing $W_{n}$.

Let now $\Pi_{n}(\cdot)$ be a homogeneous Poisson point process (h.p.p.p.), the intensity of which is equal to $n \lambda(\cdot)$.

Consider the narrowing $\Pi_{n}(A)$ of this process to the set $A$. We denote by $C_{n}^{\prime}$ the convex hull generated by it, and the set of its vertices we denote by $W_{n}^{\prime}$.

Lemma 2. The probability that at least one of the vertices $C_{n}^{\prime}$ laying in $B_{\varepsilon}$, converges to zero, as $n \rightarrow \infty$ uniformly in $\varepsilon>\varepsilon_{n}$, where $\varepsilon_{n}$, is determined by relation (1).

Proof. We assume that

$$
E^{\prime}=\left\{W_{n}^{\prime} \bigcap B_{\varepsilon} \neq \varnothing\right\}
$$


and let $\mu_{n}(\cdot)$ be the random counting measure corresponding to $\Pi_{n}(A)$. By the formula of total probability we have

$$
P\left(E^{\prime}\right)=\sum_{k=0}^{\infty} P\left(\mu_{n}(A)=k\right) P\left(E^{\prime} / \mu_{n}(A)=k\right) .
$$

Since the conditional distribution $\Pi_{n}(A)$ under the condition $\mu_{n}(A)=k$ coincides with $B_{n}(A)$, according to Lemma 1 for $k \geqslant 3$ we have

$$
P\left(E^{\prime} / \mu_{n}(A)=k\right) \leqslant k^{2}(1-c(\varepsilon))^{k-2} .
$$

Taking into account (2) and (3), we write

$$
P\left(E^{\prime}\right) \leqslant \sum_{|k-n|<\frac{n}{4}} k^{2}(1-c(\varepsilon))^{k-2} P\left(\mu_{n}(A)=k\right)+P\left(\left|\mu_{n}(A)-n\right| \geqslant \frac{n}{4}\right)=\Sigma_{1}+\Sigma_{2} .
$$

Using the Chebyshev inequality, we have

$$
\Sigma_{2} \leqslant 16 n^{-1}
$$

Further on, for sufficiently small $\varepsilon>0$

$$
\Sigma_{1} \leqslant \max _{|k-n|<\frac{n}{4}} k^{2}(1-c(\varepsilon))^{k-2} \leqslant\left(\frac{3 n}{4}\right)^{2}(1-c(\varepsilon))^{\frac{3 n}{4}-2}
$$

It is easy to see that

$$
\sup _{\varepsilon \geqslant \varepsilon_{n}} \Sigma_{1}=o(1)
$$

Combining (4)-(6), we arrive at the assertion of the lemma being proved. The lemma is proved.

Let $C_{\varepsilon}$ be the convex hull constructed from the part of the sample $X_{n}$ in $A_{\varepsilon}$.

Lemma 1 implies that

$$
\sup _{\varepsilon \geqslant \varepsilon_{n}} P\left(C_{n} \neq C_{\varepsilon}\right) \rightarrow 0 \text { as } n \rightarrow \infty
$$

Let $B_{n}\left(A_{\varepsilon}\right)$ be the narrowing of the h.b.p.p. $B_{n}(\cdot)$ on $A_{\varepsilon}$. According to Lemma 2.2 in [7], $\Pi_{n}\left(A_{\varepsilon}\right)$ and $B_{n}\left(A_{\varepsilon}\right)$ can be defined on one probability space in such a way that

$$
P\left(\Pi_{n}\left(A_{\varepsilon}\right) \neq B_{n}\left(A_{\varepsilon}\right)\right) \leqslant 2 \lambda\left(A_{\varepsilon}\right) .
$$

Let us denote the convex hull generated by $\Pi_{n}\left(A_{\varepsilon}\right)$ by $C_{\varepsilon}^{\prime}$. Then from Lemma 2 it follows that

$$
\lim _{n \rightarrow \infty} \sup _{\varepsilon \geqslant \varepsilon_{n}} P\left(C_{n}^{\prime} \neq C_{\varepsilon}^{\prime}\right)=0
$$

From (7)-(9) it follows that as $n \rightarrow \infty$

$$
P\left(C_{n}^{\prime} \neq C_{\varepsilon}^{\prime}\right) \rightarrow 0
$$

Remark. Let $f_{i}, i=1,2, \ldots, k$ be a certain finite number of functionals defined on the set of convex polygons. If the joint distribution of random variables $f_{i}\left(C_{n}\right), i=1,2, \ldots, k$ converges to some distribution $G$, then it follows from (10) that $f_{i}\left(C_{n}^{\prime}\right), i=1,2, \ldots, k$ also has this property. Thus, the problem of the limit distribution of the functionals $\nu_{n}$ and $S_{n}$, introduced in Section 1 , is reduced to the study of $\nu_{n}^{\prime}$ and $S_{n}^{\prime}$ are the corresponding characteristics of convex hulls generated by the h.p.p.p. 


\section{Convex hulls generated by the h.p.p.p.}

3.1. Some properties of the h.p.p.p. Let $K$ be a cone formed by two rays $l_{i}=$ $=\left(z: z=t e_{i}, t>0\right), i=1,2$, where $e_{1}$ and $e_{2}$ are the unit vectors. Without loss of generality, we assume that $e_{1}$ and $e_{2}$ are the orthonormal vectors

$$
e_{0}=\frac{e_{1}+e_{2}}{2}
$$

Let further $\Pi(\cdot)$ be a h.p.p.p. with intensity $\lambda(\cdot)$. We denote the narrowing on $K$ by $\Pi(K)$. Consider the convex hull $C^{\prime}$ generated by $K$ by $\Pi(K)$ and the set of its vertices $Z$.

Let us denote the vertex by $z_{0} \in Z$ for which $\left(e_{0}, z-z_{0}\right) \geqslant 0$ for all $z \in Z$.

It is obvious that $z_{0}$ is determined unambiguously almost sure.

The straight line

$$
\left(e_{0}, z-z_{0}\right)=0
$$

is the supporting line for $C^{\prime}$.

Consider a triangle formed by rays $l_{i}, i=1,2$ and a supporting line (12). We denote the set of interior points of this triangle by $\delta_{0}$, and the area is denoted by $\xi_{0}$. It is easy to see that

$$
\xi_{0}=\frac{x_{0}^{2}}{2}
$$

where $x_{0}=y_{0}=u_{0}+v_{0}$ and $z_{0}=\left(u_{0}, v_{0}\right)$. Assume that

$$
\eta_{0}=\frac{v_{0}}{x_{0}}
$$

Then from (13) and (14) it is easy to obtain

$$
u_{0}=\left(1-\eta_{0}\right) \sqrt{2 \xi_{0}}, \quad v_{0}=\eta_{0} \sqrt{2 \xi_{0}} .
$$

Let us label the vertices $C^{\prime}$, going around the boundary counterclockwise. Since $z_{0}$ is defined, each of the vertices gets its own number $j,-\infty<j<\infty$. Let us choose on the ray $l_{1}$ a sequence of points $x_{j}, j \geqslant 1$, lying on the intersection of $l_{1}$ and the lines passing through the vertices $z_{j-1}$ and $z_{j}$, respectively. Likewise, on the ray $l_{2}$, points $y_{j}, j \leqslant-1$, are obtained as a result of intersections of $l_{2}$ and the lines passing through $z_{j}, z_{j+1}$, respectively.

Let $\delta_{j}, j \neq 0$; the set of interior points of a triangle with vertices $z_{j-1},\left(x_{j-1}, 0\right),\left(x_{j}, 0\right)$, if $j \geqslant 1$, and vertices $z_{j+1},\left(0, y_{j+1}\right),\left(0, y_{j}\right)$, if $j \leqslant-1$. We denote the vertices of the triangle by $\left(x_{0}, 0\right),\left(0, y_{0}\right)$, the set of interior points by $\delta_{0}$. The third vertex of this triangle is the point $(0,0)$. The figures are taken from [6] (see Fig. 1).

We assume that

$$
\xi_{j}=\lambda\left(\delta_{j}\right)
$$

Then it is easy to obtain

$$
\xi_{j}=\left\{\begin{array}{c}
v_{j-1}\left(x_{j}-x_{j-1}\right) / 2, \text { if } j \geqslant 1 \\
u_{j+1}\left(y_{j}-y_{j+1}\right) / 2, \text { if } j \leqslant-1
\end{array},\right.
$$

where $z_{j}=\left(u_{j}, v_{j}\right)$. If we assume that

$$
\rho_{j}=\frac{u_{j}-u_{j-1}}{v_{j-1}-v_{j}}
$$




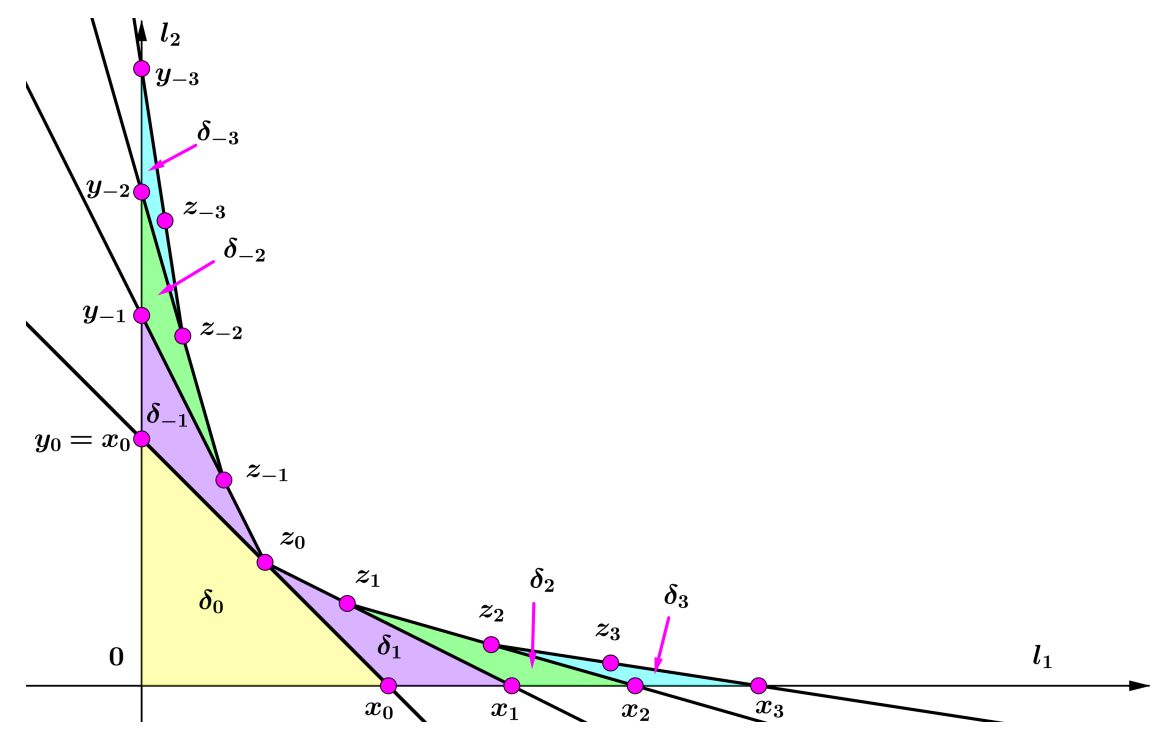

Fig. 1. Illustration of $z_{j}$ and $\delta_{j}$

then

$$
\xi_{j}=\frac{v_{j-1}^{2}}{2}\left(\rho_{j}-\rho_{j-1}\right) .
$$

Now we define the boundary functionals

$$
\theta_{T}=\inf \left\{j: x_{j} \geqslant T\right\} \text { and } \theta_{T}^{\prime}=\inf \left\{-j: y_{j} \geqslant T\right\}
$$

where $T>0$.

We assume that

$$
\begin{gathered}
\alpha(T)=\frac{2 \log T}{3}, \beta^{2}(t)=\frac{10 \log t}{27} . \\
S_{T}=\left\{\begin{array}{ll}
\xi_{1}+\xi_{2}+\ldots+\xi_{\theta_{T}} & \text { if } \theta_{T} \geqslant 1 \\
0 & \text { if } \theta_{T}=0
\end{array} \text { and } S_{T}^{\prime}=\left\{\begin{array}{ll}
\xi_{-1}+\xi_{-2}+\ldots+\xi_{-\theta_{T}^{\prime}} & \text { if } \theta_{T}^{\prime} \geqslant 1 \\
0 & \text { if } \theta_{T}^{\prime}=0
\end{array} .\right.\right.
\end{gathered}
$$

We present the following theorem with corollaries obtained in [6], which play the key role in this article (see Theorem 1, Corollaries 1, 2, 3 [6]).

Theorem 2 (Formanov and Khamdamov). Under our assumptions, as $T \rightarrow \infty$, we have

$$
(\beta(T))^{-1}\left(\theta_{T}-\alpha(T), S_{T}-\alpha(T)\right) \stackrel{d}{\Rightarrow} N(0, B) \text { with } B=\left(\begin{array}{cc}
1 & 1 \\
1 & 14 / 5
\end{array}\right) .
$$

Here $N(0, B)$ is a normally distributed random vector with a zero vector of mean values and a covariance matrix $B$.

Corollary 1 (Formanov and Khamdamov). In our case $E \theta_{T}=\alpha(T)+o(\beta(T))$ and $\operatorname{Var} \theta_{T}=$ $\beta^{2}(T)(1+o(1))$ as $T \rightarrow \infty$.

Corollary 2 (Formanov and Khamdamov). Let $0<T_{1} \leqslant T_{2}$ such that $c_{1} T_{1}<T_{2}<c_{2} T_{1}$ for some $c_{1}>0, c_{2}>0$. Then $\theta_{T_{2}}-\theta_{T_{1}}=o_{p}\left(\beta\left(T_{1}\right)\right)$ as $T_{1} \rightarrow \infty$.

Corollary 3 (Formanov and Khamdamov). Let $0<T_{1} \leqslant T_{2}$ such that $c_{1} T_{1}<T_{2}<c_{2} T_{1}$ for some $c_{1}>0, c_{2}>0$. Then $\left(S_{T_{2}}-S_{T_{1}}\right) / \beta\left(T_{1}\right)$ converges in probability to zero as $T_{1} \rightarrow \infty$. 
It is easy to see that at $\min \left\{T_{1}, T_{2}\right\} \rightarrow \infty$ the random vectors $\left(\theta_{T_{1}}, S_{T_{1}}\right)$ and $\left(\theta_{T_{2}}^{\prime}, S_{T_{2}}^{\prime}\right)$ are asymptotically independent. Moreover, the statements of Theorem 2 and its Corollaries 1-3 hold for $\left(\theta_{T_{2}}^{\prime}, S_{T_{2}}^{\prime}\right)$.

\section{Proof of Theorem 1}

The reasoning here is completely elementary. Generally, a verbal description of geometric objects is somewhat lengthy.

In accordance with the conclusions obtained at the end of Section 2 from Lemmas 1 and 2, it is sufficient to obtain the limit distribution for the number of vertices $\nu_{n}^{\prime}$ and the area $S_{n}^{\prime}$ of the convex hull $C_{n}^{\prime}$ generated by the narrowing of the $\Pi_{n}(A)$ h.p.p.p. $\Pi_{n}(\cdot)$ on the set $A$. The scheme of further reasoning is as follows. First, we divide the boundary $C_{n}^{\prime}$ into $2 r$ conditionally independent parts in such a way that each of the $r$ angles of the polygon $A$ corresponds to two elements of this partition. Thus, each of the functionals of interest to us $\nu_{n}^{\prime}$ and $S_{n}^{\prime}$ is represented as a sum of $2 r$ random variables. Then, using the properties of the h.p.p.p. stated in Section 3, the asymptotic independence and normality of these random variables are established.

Thus, the general principles for studying the problem are the same as in [7], although their implementation is completely different.

4.1. Dividing the boundary into conditionally independent parts. We denote the vertices of an $r$-gon of the support $A$ of the initial uniform distribution by $a^{(i)}, i=1,2, \ldots, r$. Let further, for some $\varepsilon>0$

$$
B_{i}=A \bigcap S\left(a^{(i)}, \varepsilon\right)
$$

where $S(z, \varepsilon)$ is a disk of radius $\varepsilon$ centered at $z$. Let us denote the narrowing $\Pi_{n}(\cdot)$ to a cone $K_{i}$ with the vertex $a^{(i)}$ and generating rays $l_{i 1}$ and $l_{i 2}$ by $\Pi_{n i}(\cdot), i=1,2, \ldots, r$, passing through $a^{(i+1)}$ and $a^{(i-1)}$ respectively. It is clear that $a^{(-1)}=a^{(r)}, a^{(r+1)}=a^{(1)}$.

Let $e_{0 i}$ play the same role with respect to $K_{i}$ as played by the vector with respect to $K_{i}$ in Section 3 . Note that $e_{0}$ is determined by the equality (11). More precisely,

$$
e_{0 i}=2^{-1}\left(\frac{a^{(i+1)}-a^{(i)}}{\left\|a^{(i+1)}-a^{(i)}\right\|}+\frac{a^{(i-1)}-a^{(i)}}{\left\|a^{(i-1)}-a^{(i)}\right\|}\right) .
$$

We denote the convex hull as $C_{n i}$ generated by $\Pi_{n i}(\cdot)$. Let us agree to denote the set of vertices $C_{n}^{\prime}$ by $Z_{n i}$. Recall that the set of vertices $C_{n}^{\prime}$ in Section 2 is denoted by $W_{n}^{\prime}$. We select in $Z_{n i}$ and $W_{n}^{\prime}$ the elements $z_{0 i}$ and $w_{0 i}$, that possess the property that the straight lines $\left(e_{0 i}, w-z_{0 i}\right)=0$ and $\left(e_{0 i}, w-w_{0 i}\right)=0$ are the supporting lines for $C_{n i}$ and $C_{n}^{\prime}$, respectively.

Assume that

$$
\Upsilon_{1}=\left\{\pi: z_{0 i}=w_{0 i}, i=1,2, \ldots, r\right\}
$$

and

$$
\Upsilon_{2}=\left\{\pi: z_{0 i} \in B_{i}, i=1,2, \ldots, r\right\},
$$

where $\pi$ is the implementation of $\Pi_{n}(\cdot)$, and $B_{i}$ is determined by equality (21).

It is easy to understand that as $n \rightarrow \infty$

$$
P\left(\Upsilon_{i}\right) \rightarrow 1, \quad i=1,2
$$


As follows from (22)-(24), with probability close to 1, the boundary of each hull $C_{n i}$ has a non-empty intersection with $C_{n}^{\prime}$. Note that the points $w_{0 i}, i=1,2, \ldots, r$ divide the boundary $C_{n}^{\prime}$ into $r$ parts. We split each of them into two more parts. Let $w^{(i)}$ be the vertex $W_{n}^{\prime} \subset C_{n}^{\prime}$, for which the straight line $\left(p_{i}, w-w^{(i)}\right)=0$, where $p_{i} \perp\left(a^{(i+1)}-a^{(i)}\right)$ is a supporting line to $C_{n}^{\prime}$. It is easy to see that $w^{(i)}$ is the closest vertex to the ray $l_{i 1}$ from the vertices $W_{n}^{\prime}$. Note that as the $n$ vertex $w^{(i)}$ grows, it approaches this ray indefinitely, i.e., $\left(p_{i}, w^{(i)}-a^{(i)}\right) \rightarrow 0$. Since the conditional distribution on the section of the supporting line $\left(p_{i}, w-w^{(i)}\right)=0$ lying in $A$, under the condition $\left(p_{i}, w^{(i)}-a^{(i)}\right)=t$ is uniform, we have

$$
\lim _{\varepsilon \rightarrow 0} \lim _{n \rightarrow 0} \inf P\left(w^{(i)} \in \bigcap_{j=1}^{r} \bar{B}_{j}\right)=1 .
$$

Hence it follows that

$$
\lim _{\varepsilon \rightarrow 0} \lim _{n \rightarrow 0} \inf P\left(\bar{w}_{i} \in \bigcap_{j=1}^{r} \bar{B}_{j}\right)=1,
$$

where $\bar{w}_{i}$ is the base of the perpendicular drawn from $w_{i}$ to $l_{i 1}$.

Consider

$$
\Upsilon_{3}=\left\{\bar{w}_{i} \in \bigcap_{j=1}^{r} \bar{B}_{j}, i=1,2, \ldots, r\right\} .
$$

As follows from (25) and (26), for any $\varepsilon>0$ one can find such $N>0$ that, for all sufficiently large $n>N$, the following inequality holds

$$
P\left(\Upsilon_{3}\right) \geqslant 1-\varepsilon .
$$

In what follows, without specifying, we consider only those implementations of $\Pi_{n}(\cdot)$ that are contained in $\bigcap_{j=1}^{3} \Upsilon_{j}$. For such implementations $w^{(i)}, i=1,2, \ldots, r$ lie between $w_{0 i}$ and $w_{0(i+1)}$. Thus, the boundary $C_{n}^{\prime}$ is divided into $2 r$ parts. It is easy to see, that these parts are conditionally independent for the given $w_{0 i}, w^{(i)}, i=1,2, \ldots, r$.

4.2. Choice of approximating functionals. Let us consider the section of the boundary $C_{n}^{\prime}$ between the vertices $w_{01}$ and $w^{(i)}$. The section between $w^{(r)}$ and $w_{01}$ is studied in a similar way. Let us label the vertices $C_{n}^{\prime}$, going around the boundary counterclockwise, starting from $w_{01}$. As a result, on the considered section of the boundary, we obtain $w_{j}, j=0,1,2, \ldots, \mu$, where $w_{0}=w_{01}, w_{\mu}=w^{(1)}$. We perform a similar operation with the vertices $z \in C_{n 1}^{\prime}$, obtaining $z_{j}, j=0,1,2, \ldots$, where, in view of (22) and (24) $z_{0}=w_{01}=w_{0}$.

In order to use the h.p.p.p. properties described in Section 3, we need to proceed from $\Pi(\cdot)$ to $\Pi_{n}(\cdot)$. In such transition, the linear characteristics $x_{j}, y_{j}, u_{j}, v_{j}$, change to $x_{j}^{\prime}=n^{-\frac{1}{2}} x_{j}$, $y_{j}^{\prime}=n^{-\frac{1}{2}} y_{j}, u_{j}^{\prime}=n^{-\frac{1}{2}} u_{j}, v_{j}^{\prime}=n^{-\frac{1}{2}} v_{j}$ respectively, while the area $\xi_{j}$ of the triangle $\delta_{j}$ becomes $\xi_{j}^{\prime}=n^{-1} \xi_{j}$. Dimensionless quantities $\eta_{j}, \tau_{j}, \rho_{j}$ remain unchanged in such transition. We denote the images $z_{j}$ of such a transformation by $z_{j}^{\prime}$.

Let $T=\varepsilon \sqrt{n}, T_{1}=h \sqrt{n}$ where $h$ is the length of the side $A$ connecting the vertices $a^{(1)}$ and $a^{(2)}$. In accordance with (19), we assume that

$$
\theta=\theta_{T} \text { and } \chi=\theta_{T_{1}}
$$

It is clear that

$$
\theta=\inf \left\{j: x_{j}^{\prime} \geqslant \varepsilon\right\} \text { and } \chi=\inf \left\{j: x_{j}^{\prime} \geqslant h\right\}
$$


Note that $x_{j}$ and $x_{j}^{\prime}$ are constructed on the vertices $z_{j-1}, z_{j}$ and $z_{j-1}^{\prime}, z_{j}^{\prime}$, respectively. Note that $w_{j}=z_{j}^{\prime}$, at least for $0 \leqslant j \leqslant \chi-1$.

Let further

$$
p=\xi_{1}^{\prime}+\xi_{2}^{\prime}+\cdots+\xi_{\theta}^{\prime},
$$

and

$$
q=\xi_{1}^{\prime}+\xi_{2}^{\prime}+\cdots+\xi_{\chi}^{\prime}
$$

Assume that

$$
\theta^{*}=\frac{\theta-\alpha}{\beta_{1}}, \quad p^{*}=\frac{n p-\alpha}{\beta_{2}}
$$

where

$$
\alpha=\frac{1}{3} \log n, \quad \beta_{1}=\sqrt{\frac{5 \log n}{27}}, \quad \beta_{2}=\sqrt{\frac{14 \log n}{27}} .
$$

From (20), (27), (28) and Theorem 2 it follows that

$$
\left(\theta^{*}, p\right) \stackrel{d}{\Rightarrow} \omega
$$

where $\omega$ is determined from Theorem 1 . Now we assume that

$$
\chi^{*}=\frac{\chi-\alpha}{\beta_{1}}, \quad q^{*}=\frac{n q-\alpha}{\beta_{2}} .
$$

According to Corollaries 1-3, in view of (28) and (30), we have

$$
\frac{\theta-\chi}{\beta_{1}} \stackrel{p}{\rightarrow} 0, \quad \frac{n(p-q)}{\beta_{1}} \stackrel{p}{\rightarrow} 0 .
$$

From (29)-(33) follows that

$$
\left(\chi^{*}, q^{*}\right) \stackrel{d}{\Rightarrow} \omega .
$$

Similar characteristics $\theta^{\prime}, p^{\prime}$ and $\chi^{\prime}, q^{\prime}$ constructed along the section of the boundary $C_{n}^{\prime}$ between the vertices $w^{(r)}$ and $w_{01}=w$, also have properties (31) and (34). It is important that they are asymptotically independent of $\theta, \chi, p$ and $q$. And no less important is the fact that $\theta, \theta^{\prime}, p$ and $p^{\prime}$ are completely determined by the narrowing of $\Pi_{n}(\cdot)$ to $B_{1}$. It follows that similar characteristics $\theta_{i}, \theta_{i}^{\prime}, p_{i}, p_{i}^{\prime}$ for the boundary sections corresponding to the angles with the vertices $a^{(i)}, i=1,2, \ldots, r$ are independent. By analogy with (29) and (32), we define

$$
\Theta^{*}=\frac{\Theta-2 r \alpha}{\beta_{1} \sqrt{2 r}} \text { and } P^{*}=\frac{n P-2 r \alpha}{\beta_{2} \sqrt{2 r}},
$$

where

$$
\Theta=\sum_{i=1}^{r}\left(\theta_{i}+\theta_{i}^{\prime}\right), \quad P=\sum_{i=1}^{r}\left(p_{i}+p_{i}^{\prime}\right) .
$$

Due to independence of $\left(\theta_{i}+\theta_{i}^{\prime}, p_{j}+p_{j}^{\prime}\right), i, j-1,2, \ldots, r$, from (31) we obtain

$$
(\Theta, P) \stackrel{d}{\Rightarrow} \omega .
$$

Finally, by analogy with (35), we introduce

$$
\mathbb{X}^{*}=\frac{\mathbb{X}-2 r \alpha}{\beta_{1} \sqrt{2 r}}, \quad \mathbb{Q}^{*}=\frac{\mathbb{Q}-2 r \alpha}{\beta_{2} \sqrt{2 r}},
$$


where (compare with (35))

$$
\mathbb{X}=\sum_{i=1}^{r}\left(\chi_{i}+\chi_{i}^{\prime}\right), \quad \mathbb{Q}=\sum_{i=1}^{r}\left(q_{i}+q_{i}^{\prime}\right) .
$$

Note that $\left(\chi_{i}+\chi_{i}^{\prime}, q_{i}+q_{i}^{\prime}\right), i=1,2, \ldots, r$, generally speaking, are independent. However, in view of (33) and (34), we can assert that

$$
\left(\mathbb{X}^{*}, \mathbb{Q}^{*}\right) \stackrel{d}{\Rightarrow} \omega
$$

It is the functionals $\mathbb{X}^{*}$ and $\mathbb{Q}^{*}$ that give us the required approximation for $\nu_{n}^{\prime}$ and $S_{n}^{\prime}$.

4.3. Estimation of the approximation accuracy. Let $s$ be the area of the figure bounded by the section of the boundary $C_{n}^{\prime}$ between the vertices $w_{0}=w_{01}$ and $w_{\mu}=w^{(1)}$, the segment of the ray $l_{11}$ between the points $\bar{w}_{1}$ and $x_{0}^{\prime} e_{11}$ and the supporting line $\left(e_{01}, w-w_{01}\right)=0$. Here, the points $w_{0}, w_{\mu}, \bar{w}_{1}$ are defined in Sections 4.1 and 4.2 ,

$$
e_{11}=\frac{a^{(2)}-a^{(1)}}{\left\|a^{(2)}-a^{(1)}\right\|}
$$

and $x_{0}^{\prime}$ corresponds to $x_{0}$ when going from $\Pi(\cdot)$ to $\Pi_{n}(\cdot)$.

Let us construct similar left characteristics $\mu^{\prime}$ and $s^{\prime}$ in the section of the boundary between the vertices $w^{(r)}$ and $w_{01}$.

In what follows, we denote $\mu_{i}, \mu_{i}^{\prime}, s_{i}$ and $s_{i}^{\prime}$, the analogs of $\mu, \mu^{\prime}, s$ and $s^{\prime}$, corresponding to the angle with the vertex $a^{(i)}$. It is easy to see that $\nu_{n}^{\prime}$ is the total number of vertices $C_{n}^{\prime}$ and can be represented as

$$
\nu_{n}^{\prime}=\sum_{i=1}^{r}\left(\mu_{i}+\mu_{i}^{\prime}\right) .
$$

And area $A-C_{n}^{\prime}$ can be represented in the form

$$
\lambda\left(A-C_{n}^{\prime}\right)=\sum_{i=1}^{r}\left(s_{i}+s_{i}^{\prime}\right)+\xi_{0 i}^{\prime},
$$

where $\xi_{0 i}^{\prime}$ is the area of the triangle cut by the supporting line $\left(e_{0 i}, w-w_{0 i}\right)=0$.

Note that

$$
n \xi_{01}^{\prime}=\xi_{0}=O(1)
$$

where $\xi_{0}$ has an exponential distribution (see for example [6]). Similarly

$$
n \xi_{0 i}^{\prime}=\xi_{0}=O(1), i=1,2, \ldots, r
$$

As an approximation for $\mu_{i}, \mu_{i}^{\prime}, s_{i}$ and $s_{i}^{\prime}$, we use $\chi_{i}, \chi_{i}^{\prime}, q_{i}$ and $q_{i}^{\prime}$, introduced in Section 4.2. In this case, it is enough to evaluate the proximity of $\left(\mu_{1}, s_{1}\right) \stackrel{\text { dis }}{=}(\mu, s)+o_{p}(1)$ and $\left(\chi_{1}, q_{1}\right) \stackrel{\text { dis }}{=}(\chi, q)$. The remaining pairs of vectors are matched similarly.

To complete the proof of the theorem, it suffices to show the proximity of $s$ and $q$, i.e.

$$
\frac{n(s-q)}{\sqrt{\log n}} \stackrel{p}{\rightarrow} 0 \text { at } n \rightarrow \infty
$$

and proximity of $\mu$ and $\chi$, i.e.

$$
\frac{\mu-\chi}{\sqrt{\log n}} \stackrel{p}{\rightarrow} 0 \text { at } n \rightarrow \infty .
$$


We obtain the relation (42) from Corollary 3, and relation (43) from Corollary 2. The obtained relations (42) and (43) with the relations (36), (37)-(41) allow us to assert that a random vector with components $\frac{\nu_{n}^{\prime}-2 r \alpha}{\beta_{1} \sqrt{2 r}}$ and $\frac{n\left(1-S_{n}^{\prime}-2 r \alpha\right.}{\beta_{2} \sqrt{2 r}}$ converges in distribution to $\omega$. Taking into account Remark given at the end of Section 3, we obtain the assertion of the theorem. The theorem is proved.

\section{References}

[1] C.Buchta, On the distribution of the number of vertices of a random polygon, Anz. Osterreich. Akad. Wiss. Math. Natur. K1, 139(2003), 17-19.

[2] C.Buchta, Exact formulae for variances of functionals of convex hulls, Advances in Applied Probability, 45(2013), no. 4, 917-924. DOI: 10.1239/aap/1386857850

[3] A.J.Cabo, P.Groeneboom, Limit theorems for functiohals of convex hulls, Probab. Theory Relat. Fields, 100(1994), 31-55.

[4] H.Carnal, Die konvexe Hulle von $\mathrm{n}$ rotations symmetrisch verteilten Punkten, $Z$. Wahrscheinlichkeits theorie verw. Geb., (1970), no. 15, 168-176.

[5] B.Efron, The convex hull of a random set of points. Biometrika, 52(1965), 331-343.

[6] Sh.K.Formanov, I.M.Khamdamov, On joint probability distribution of the number of vertices and area of the convex hulls generated by a Poisson point process, Statistics and Probability Letters, 169(2021), 108966, 1-7.

[7] P.Groeneboom, Limit theorems for convex hulls, Probab. Theory Related Fields, 79(1988), $327-368$.

[8] P.Groeneboom, Convex hulls of uniform samples from a convex polygon, Adv. Appl. Prob. (SGSA), 44(2012), 330-342. DOI: 10.1239/aap/1339878714

[9] T.Hsing, On the asymptotic distribution of the area outside a random convex hull in a disk, The Annals of Applied Probability, 4(1994), no. 2, 478-493.

[10] I.M.Khamdamov, On Limit Theorem for the Number of Vertices of the Convex Hulls in a Unit Disk, Journal of Siberian Federal University. Mathematics and Physics, 13(2020), no. 3, 275-284. DOI: 10.17516/1997-1397-2020-13-3-275-284

[11] I.M.Khamdamov, Properties of convex hull generated by inhomogeneous Poisson point process, Ufa Mathematical Journal, 12(2020), no. 3, 81-96. DOI: 10.13108/2020-12-3-81

[12] A.V.Nagaev, Some properties of convex hulls generated by homogeneous Poisson point processes in an unbounded convex domain, Ann. Inst. Statist. Math., 47(1995), no. 1, 21-29.

[13] A.V.Nagaev, I.M.Khamdamov, Limit theorems for functionals of random convex hulls. Preprint of Institute of Mathematics, Academy of Sciences of Uzbekistan, Tashkent, 1991 (in Russian.)

[14] J.Pardon, Central limit theorems for random polygons in an arbitrary convex set, The Annals of Probability, 1(2011), no. 3, 881-903. 
[15] J.Pardon, Central Limit Theorems for Uniform Model Random Polygons, J. Theor. Probab, 25(2012), 823-833.

[16] A.Reny, R.Sulanke, Uber diekovexe Hulle von zufalling gewahlten Punkte, Z. Wahrscheinlichkeits theorie verw. Geb., 2(1963), 75-84.

\title{
Совместное распределение числа вершин и площади выпуклых оболочек, порожденных равномерным распределением в выпуклом многоугольнике
}

\author{
Исакжан М. Хамдамов \\ Национальный университет Узбекистана им. Мирзо Улугбека \\ Ташкент, Узбекистан \\ Зоя С. Чай \\ Ташкентский университет информационных технологий
}

Ташкент, Узбекистан

\begin{abstract}
Аннотация. Рассматривается выпуклая оболочка, порожденная выборкой, равномерно распределенной на плоскости для случая, когда носитель распределения представляет собой выпуклый многоугольник. Доказывается центральная предельная теорема для совместного распределения числа вершин и площади выпуклой оболочки с использованием пуассоновской аппроксимации биномиальных точечных процессов вблизи границы носителя распределения. Здесь применяются результаты [6] совместного распределения числа вершин и площади выпуклых оболочек, порожденных пуассоновским распределением. Из результатов, полученных в настоящей статье, в частности, следуют результаты [3,7], когда носитель представляет собой выпуклый многоугольник, а выпуклая оболочка порождается однородным пуассоновским точечным процессом.
\end{abstract}

Ключевые слова: выпуклая оболочка, выпуклый многоугольник, пуассоновский точечный процесс, биномиальный точечный процесс, центральная предельная теорема. 